\title{
TRPC1 expression and distribution in rat hearts
}

\author{
H. Huang, W. Wang, P. Liu, Y. Jiang, Y. Zhao, H. Wei, W. Niu \\ ${ }^{1}$ Department of Physiology, Capital Medical University, Beijing, China
}

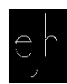

(C)2009 European Journal of Histochemistry

Transient receptor potential canonical (TRPC) proteins have been identified as a family of plasma membrane calcium-permeable channels. TRPC proteins can be activated by various stimuli and act as cellular sensors in mammals. Stretch-activated ion channels (SACs) have been proposed to underlie cardiac mechano-electric feedback (MEF), although the molecular entity of SAC remains unknown. There is evidence suggesting that transient receptor potential canonical 1 (TRPC1) is a stretch-activated ion channel. As a non-selective cation channel, TRPC1 may cause stretch-induced depolarization and arrhythmia and thus may contribute to the MEF of the heart. In this study, we examined the expression patterns of TRPC1 in detail at both the mRNA and protein levels in rat hearts. We isolated total RNA from the left and right atria, and the left and right ventricles, and detected TRPC1 mRNA in these tissues using reverse-transcriptase polymerase chain reaction (RT-PCR). To study the protein localization and targeting, we performed immunohistochemistry and immunofluorescence labeling with the antibody against TRPC1. TRPC1 was detected in the cardiomyocytes of the ventricle and atrium at both the mRNA and protein levels. The cell membrane and Ttubule showed strong fluorescence labeling in the ventricular myocytes. Purkinje cells, the endothelial cells and smooth muscle cells of the coronary arterioles also displayed TRPC1 labeling. No TRPC1 was detected in fibroblasts. In conclusion, TRPC1 is widely expressed in the rat heart, including in working cells, Purkinje cells and vascular cells, suggesting that it plays an important role in the heart. The specific distribution pattern offered a useful insight into its function in adult rat ventricular cells. Further investigations are needed to clarify the role of TRPC1 in regulating cardiac activity, including cardiac MEF.

Key words: TRPC1, Heart, Expression and distribution.

Correspondence: Weizhen Niu,

Department of physiology, Capital Medical University, You An Men Wai Street Xitoutiao 10, Beijing 100069, China Tel: +86.10.83911470.

E-mail: niuwz@yahoo.cn

Paper accepted on September 9, 2009

European Journal of Histochemistry 2009; vol. 53 issue 4 (October-December): 217-224

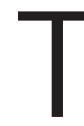
he heart not only functions as a pump but also senses the variational stress on itself during the cardiac cycle. Proper mechanical loading is necessary for the development and maturation of the heart and to maintain normal function (Tobita and Keller, 2000). However, overloading or mechanical stimulus causes cardiac hypertrophy and arrhythmias (Clemo et al., 1998; Sadoshima et al., 1992a; Schrickel et al., 2002). Numerous studies have shown that a mechanical stretch or load applied to a cardiac tissue can induce significant electrophysiological changes via the process termed "mechano-electric feedback" (MEF). The underlying mechanisms linking such a mechanical impact to subsequent arrhythmias remain unknown.

Mechanosensitive channels are proving germane to our understanding of cardiac MEF. The electrophysiological changes during MEF have been principally attributed to the activity of stretch-activated ion channels (SACs) (Hu and Sachs, 1997), whose open probability was augmented with increasing membrane tension. Two groups of SACs in the heart have been reported: stretch-activated potassium channels (SAKCs) and stretch-activated non-selective cation channels (SACCS). Recent studies suggest that the two-pore domain potassium channel TREK-1 may be the molecular entity of SAKCs in the heart ( Liu et al., 2008; Tan et al., 2004; Xian Tao et al., 2006). However, the identities of the SACCs remain unknown. SACCs transduce membrane stretch into cation $\left(\mathrm{Na}^{+}, \mathrm{K}^{+}, \mathrm{Ca}^{2+}\right.$ and $\mathrm{Mg}^{2+}$ ) fluxes across the cell membrane and are implicated in both stretch-induced depolarization and cardiac arrhythmias (Franz et al., 1992; Wei et al., 2006). Identifying the molecular components of SACCS and elucidating the mechanisms of activation are of equal importance, and both tasks have proven difficult.

In recent years, attention has been focused on the idea that the transient receptor potential (TRP) channels act as cellular sensors (Clapham, 
2003). Particularly, the TRP-related family members OSM-9, OTRPC4 and NOMPC have been implicated in osmoreception (Clapham, 2003). As non-selective cation channels, TRP channels with mechanosensitivities may play an important role in MEF. Canonical TRP (TRPC) channels are a group of mammalian $\mathrm{Ca}^{2+}$-permeable channels that share homology with the Drosophila TRP channels (Minke and Cook, 2002).TRPC channels mediate store-operated $\mathrm{Ca}^{2+}$ entry as well as store-independent $\mathrm{Ca}^{2+}$ influx (Kwan et al., 2004; Minke and Cook, 2002; Nilius et al., 2003). TRPC channels are unlikely to play an important role in relation to the $\left[\mathrm{Ca}^{2+}\right]$ i fluctuations responsible for excitation-contraction coupling. Rather, they are more likely to control local $\left[\mathrm{Ca}^{2+}\right]_{i}$, which may play a role in regulating the signaling pathways that contribute to positive inotropic responses, arrhythmias, cardiac damage following ischemiareperfusion, myocyte hypertrophy and changes in gene expression (Ohba et al., 2007).

Aside from the well-known voltage-gated calcium channels (VGCCs), TRP proteins have attracted almost exponentially growing attention as exceptionally unique non-voltage-gated $\mathrm{Ca}^{2+}$-entry channels activated by a multitude of physiochemical stimuli and associated with a diversity of biological functions. Most recently, a member of the TRPC subfamily, TRPCl, was confirmed to be mechanosensitive and to form the SACC in vertebrate cells (Maroto et al., 2005). Although another study showed that overexpression of hTRPCl in mammalian cells does not increase the SACC activities significantly, one of the reasons may be that plasma membrane TRPCl does not increase in this study (Gottlieb et al., 2008). The factors involved in regulating TRPCl trafficking as well as TRPCl channel function are complicated and worthy of further study.

Anyway, TRPCl is one of the most studied TRP channels (Wes et al., 1995; Zhu et al., 1995; Zitt et al., 1996). At the cellular level, TRPC1 functions as a pathway of non-voltage-gated $\mathrm{Ca}^{2+}$ and $\mathrm{Na}^{+}$influxes that may also include the stretchactivated cation influx. Thus, clarifying the expression and distribution of TRPCl in the heart is a necessary step to offer insight into whether or not TRPCl can act as a SACC in the heart.

Studies directly and detailedly demonstrating TRPCl localization and targeting in intact rat hearts are lacking except a few reverse-transcrip- tion polymerase chain reaction ( $R T-P C R$ ) experiments. In the present study, we have combined RT$P C R$ and immunohistochemistry to demonstrate the overall expression patterns of TRPCl in the rat heart. Our data provide the reference information both for further identification of TRPCl as potential candidate SACC in the rat heart and for further investigation of TRPCl related cardiac MEF.

\section{Materials and Methods}

\section{RT-PCR}

Total RNA was extracted from left ventricle, right ventricle, left atrium and right atrium of adult Sprague-Dawley (SD) rats $(230-250 \mathrm{~g})$ respectively, using the trizol-chloroform-isopropyl alcohol method (Invitrogen, Carlsbad, USA). RTPCR was performed using a two-step RT-PCR kit (Takara RNA PCR Kit (AMW) Ver. 3.0, Takara, Otsu, Japan). Total RNA was reversely transcribed into first-strand cDNA using oligo-dT primers and AMV reverse transcriptase (Takara, Otsu, Japan). Reverse transcription was performed at $42^{\circ} \mathrm{C}$ for 30 minutes, followed by a final terminal reaction at $99^{\circ} \mathrm{C}$ for 15 minutes. The cDNA products were used as templates for PCR amplification, which was performed with Taq DNA polymerase (Takara, Otsu, Japan). The primers for PCR were designed according to the sequence of rat TRPCl mRNA available in the GenBank database (access number: NM_053558). The primer pair (forward/reverse) was: 5'-CTC TTG ACA AAC GAG GAC TAC TA-3' (in exon 5)/ 5'-GTC TTC CAA CCC TTC ATA CCA-3' (in exon 7). Cycling conditions were as follows: 2 minutes at $94^{\circ} \mathrm{C}$ followed by 40 cycles of 30 seconds at $94^{\circ} \mathrm{C}, 30$ seconds at $55^{\circ} \mathrm{C}, 30$ seconds at $72^{\circ} \mathrm{C}$ and a final extension of 7 minutes at $72^{\circ} \mathrm{C}$. Control reactions without template RNA or the reverse transcriptase were included for each PCR amplification experiment. PCR products were separated on $1.5 \%$ agarose gels by electrophoresis and visualized by staining with ethidium bromide. The authenticity of amplified PCR products was verified using an $A B I$ PRISM DNA sequencing system (Perkin Elmer).

\section{Immunohistochemistry}

The heart of SD rat was used for immunohistochemical experiments. Immunoreactivity was tested using avidin-biotin-peroxidase reactions. Tissue 
cross-sections of $3 \mu \mathrm{m}$ were rehydrated in a graded alcohol series to $70 \%$ ethanol, washed with deionized water and then preincubated with $3 \%$ (v/v) $\mathrm{H}_{2} \mathrm{O}_{2}$ in absolute methanol in order to inhibit endogenous peroxidase activity. Normal goat serum was then used to block the endogenous biotin. Sections were incubated at $4^{\circ} \mathrm{C}$ overnight with rabbit anti-rat $\mathrm{TRPCl}$ primary antibodies (1:100 dilution, batch number AN-04, Alomone Labs, Jerusalem, Israel). Secondary biotinylated goat anti-rabbit IgG was subsequently applied, the immunoreactivity was visualized with streptavidin-biotin-peroxidase using 3, 3'-diaminobenzidine (Sigma-Aldrich, St. Louis, USA) as a substrate, and the sections were counterstained with hematoxylin to show nuclei. In negative control experiments, the primary antibodies were either omitted or were preabsorbed for 2.5 hours at room temperature with a 10-fold molar excess of peptide antigens provided by the manufacturer. A positive control was performed on skeletal muscle as the positive tissue because the presence of TRPCl in skeletal muscle had previously been confirmed (Vandebrouck et al., 2002).

\section{Immunofluorescence}

Ventricular myocytes were enzymatically isolated from adult SD rat heart, as described previously (Niu and Sachs, 2003). Cells in suspension were transferred to slides, fixed in cold $4 \%$ paraformaldehyde solution for 15 minutes, permeabilized with $0.3 \%$ Triton X-100 for 10 minutes at room temperature, and preincubated with $3 \%$ ( v/v) $\mathrm{H}_{2} \mathrm{O}_{2}$ in absolute methanol for 5 minutes. Normal goat serum was used to block endogenous biotin. Then the cells were exposed to primary (rabbit anti-rat TRPC1, 1:100 dilution, batch number AN-04, Alomone Labs, Jerusalem, Israel) and secondary (tetramethyl rhodamine isothiocyanate (TRITC)-conjugated goat anti-rabbit IgG, Jackson Labs, West Grove, USA) antibodies. Actin filaments were stained with $5 \mu \mathrm{g} / \mathrm{mL}$ of Alexa Fluor 488 phalloidin (Molecular Probes, Eugene, USA) at $4^{\circ} \mathrm{C}$ for 30 minutes. The myocytes were visualized using a confocal microsystem (LAS AF-TCS SP5, Leica, Wetzlar, Germany). Rhodamine (TRITC) was excited at $561 \mathrm{~nm}$ and detected at 585-640 nm. Alexa Fluor 488 phalloidin was excited at $495 \mathrm{~nm}$ and detected at $519 \mathrm{~nm}$.

\section{Results}

\section{RT-PCR-based detection of TRPC1 expression in rat hearts}

RT-PCR was used to examine the expression of TRPCl transcripts. Primers were designed according to the corresponding rat TRPCI mRNA sequences ( $N M$ M_053558). Forward and reverse primers for TRPCl were located in separate exons. RT-PCR amplified the expected 467 base pair (bp) product indicative of TRPCl from total RNA isolated from left ventricle, right ventricle, left atrium and right atrium of rat (Figure 1 ). The 467 bp product for TRPCl did not result from genomic DNA contamination since PCR amplification from genomic DNA should result in products with a much larger molecular size. The product was absent in the control experiment, which was performed without template RNA or reverse transcriptase (data not shown). The authenticity of the $467 \mathrm{bp}$ product was confirmed by DNA sequencing (data not shown).

\section{Detection of TRPC1 in rat hearts by immunohisto- chemistry}

Immunohistochemistry was used to explore the cellular localization of TRPCl in the rat heart. Strong positive signals, brown in color, can be observed in the cardiomyocytes of ventricles (Figure 2A) and atria (Figure 2B), particularly on the cell membrane of the ventricular myocytes. The immunohistochemical studies also confirmed positive signals in the endothelial cells and the smooth muscle layers of coronary arterioles, although the staining was much weaker than that seen in cardiomyocytes (Figure $2 \mathrm{C}$ ). Purkinje cells beneath the endocardium were also positively stained. Purkinje cells were characterized by their special shape and pigmentation via hematoxylin

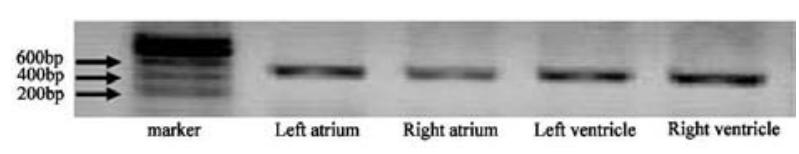

Figure 1 RT-PCR based detection of TRPC1 in rat hearts. PCR products were observed in ethidium bromide-stained agarose gel. TRPC1 DNA fragments (467 bp) were amplified from left atrium, right atrium, left ventricle and right ventricle of rats. 

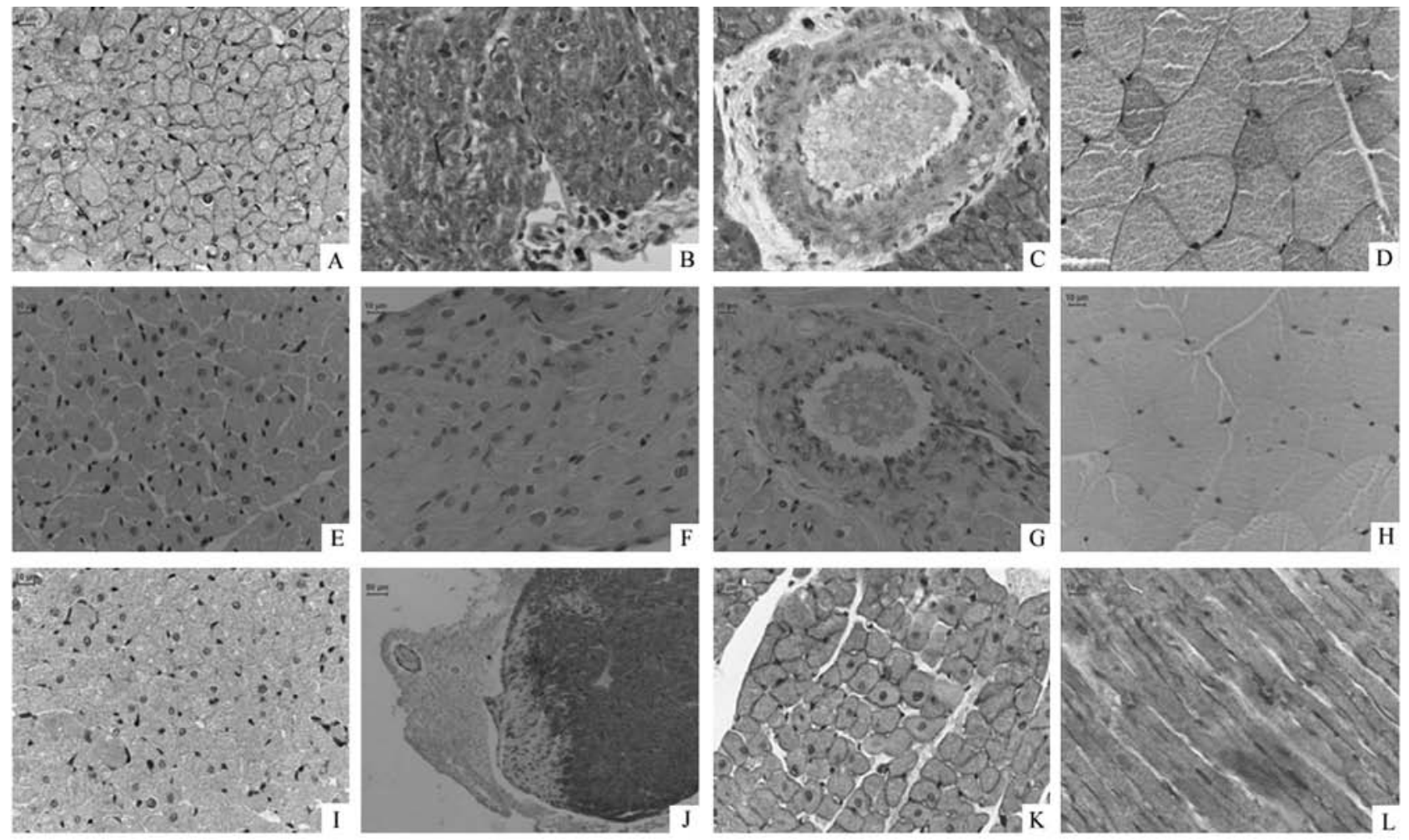

Figure 2. Immunohistochemical detection of TRPC1 protein in rat hearts. Sections were incubated with primary antibody for TRPC1 (A, B, C, D), without primary antibody (E, F, G, H) or with primary antibody preabsorbed by TRPC1 peptide for negative control (I). Positive signals in brown color can be visualized in the myocytes of the left ventricle (A) and atrium (B), endothelial and smooth muscle layers of coronary arterioles (C), and skeletal muscle cells (D, as positive control). No positive signal could be observed in control experiments without primary antibody. A faint signal was occasionally observed in antigen preabsorption control (I). There are negative cells in the edge of ventricular tissues $(J)$ and also the fibroblasts between ventricular myocytes which showed blue nuclei without positive signals. The right ventricle shows the same distribution of TRPC1 positive signal (K) as the left ventricle. TRPC1 showed intense staining on the cell membranes of ventricular myocytes (A, K, L) and skeletal muscle cells (D). The longitudinal section of left ventricle also shows striated distribution of TRPC1 (L). Scale bar $=10 \mu \mathrm{m}$, except scale bar $=50 \mu \mathrm{m}$ in panel J.
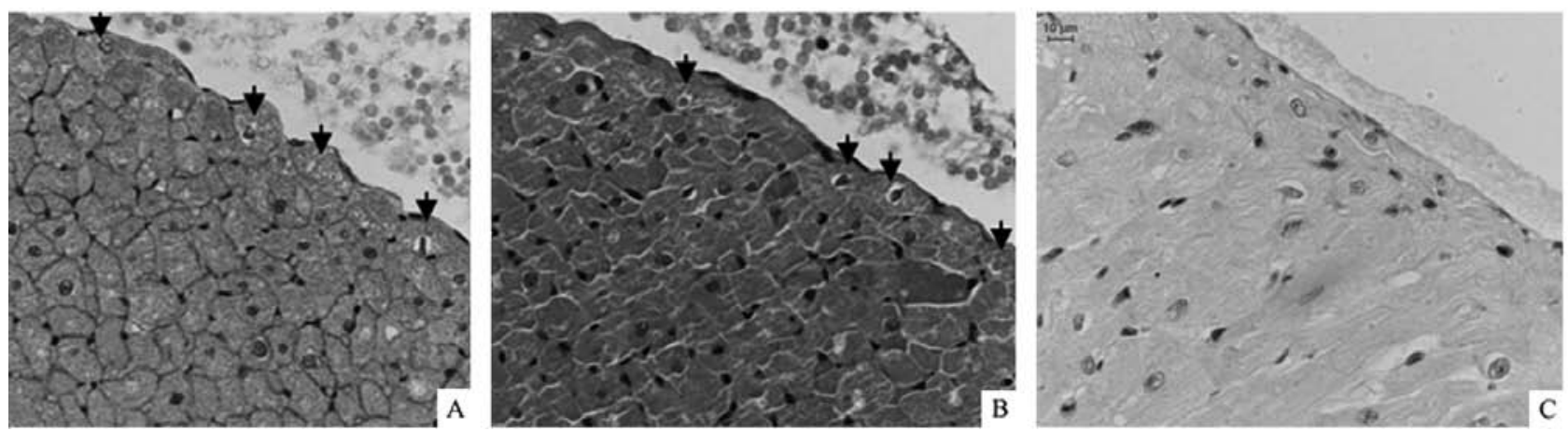

Figure 3. Distribution of TRPC1 in Purkinje cells. These sections were contiguous tissue cross-sections. Endocardial layers were shown. Positive signals, brown in color, could be visualized in Purkinje cells (A, black arrows showed Purkinje cells). The cells beneath the endocardium with loose cytoplasmic structure and without any structure around nuclei were Purkinje cells according to HE staining (B, black arrows showed Purkinje cells). No positive signal could be observed in control experiments (C). Scale bar $=10 \mu \mathrm{m}$.

and eosin (HE) staining using the tissue cross-sections contiguous to those used for immunohistochemical study (Figure 3). These results indicate a wide distribution of TRPCl in the rat hearts, including working cells, Purkinje cells, endothelial cells and smooth muscle cells of coronary arterioles. No positive signal was observed in fibroblasts. Efforts were also made to display the expression 
pattern of TRPCl in skeletal muscle as a positive control. This procedure could overcome the potential for non-specific staining during immunohistochemical experiments. Our results show that the distribution pattern of TRPCl in cardiomyocytes is similar to that in skeletal muscle. Both plasma and cell membrane were labeled with TRPCl antibodies, and the membrane had a stronger stain (Figure 2D).

Two sets of negative control experiments were performed: one with antigen (a peptide with the sequence QLYDKGYTSKEQKDC, corresponding to amino acids 557 571 of the TRPCl protein) preabsorption and the other in the absence of primary antibodies. No signal was observed in the absence of primary antibodies (Figure 2E, F, G, $H$ ). Faint signal was occasionally seen in the antigen preabsorption control, which may be due to insufficient preabsorption (Figure 2I). Nevertheless, the immunospecificity of TRPCI antibody is authentic, given the distinctively different staining between the experimental group (without preabsorption) and the control group (with preabsorption). The blue color in the images results from hematoxylin counterstaining, showing the locations of cell nuclei.

Confocal images of the ventricular and atrial myocytes stained with anti-TRPCl antibody showed the cell membrane and plasma localization of TRPC1. Alexa Fluor 488 phalloidin staining showed regular transverse striations of the I bands. We also observed a clear transverse-striation pattern of TRPCl distribution parallel to and close to the striation of the F-actin stained by phalloidin, consistent with transverse-tubular localization in the ventricular cell (Figure 4), whereas there was no such distribution in the atrial cell which lacked T-tubules.

Both RT-PCR and immunohistochemical experiments were independently repeated at least six times and all results from each repetition were consistent.

\section{Discussion}

Recently, endogenous TRPC expression (and in some cases the related protein) have been described in a variety of cell types, including vascular endothelial cells (Antoniotti et al., 2002), smooth muscle cells (Yip et al, 2004), and specif-

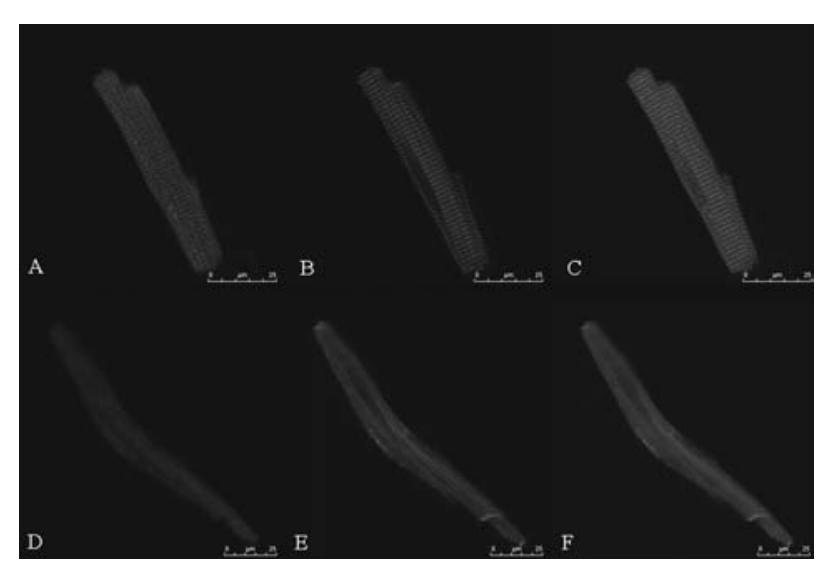

Figure 4. Localization of TRPC1 in rat cardiomyocytes shown by confocal images. Cardiac myocytes were double stained by antiTRPC1 antibody (A and D) and phalloidin (B and E). Panels C and $F$ show a merged image of panel $A / B$ and $D / E$ respectively, where TRPC1 is colored in red and phalloidin in green. The transverse striation of actin filaments can be seen both in the ventricular myocytes (B) and the atrial myocytes (E). Note that TRPC1 in the ventricular myocyte $(A)$ are parallel to and close to transverse striation of actin filaments, suggesting that they are located at T-tubules while TRPC1 in the atrial myocytes (D) do not show the striation-like distribution. Scale bar $=25 \mu \mathrm{m}$.

ic type of nervous system cells (Riccio et al, 2002). Evidence is accumulating that channels of the TRP superfamily play sensory roles in a wide variety of receptor cells, including mechanoreceptor cells ( Lin and Corey, 2005). The transduction mechanisms linking stretch and downstream events have not been fully explored, but in most cell types mechanotransduction is mediated by integrin signaling and stretch-activated cation influx (Iqbal and Zaidi, 2005; Shaw and Xu, 2003). Recent reports suggest that proteins of the TRP superfamily form mechanosensitive cation channels (Corey et al., 2004; Maroto et al., 2005). The rise of intracellular calcium in cardiac myocytes and vascular smooth muscle cells may be mediated also via stretch-activated channels (Calaghan et al., 2003; Liao et al., 2003; Zou et al., 2002) besides release of intracellular calcium stores and influxes through L-type cation channel and sodium-calcium exchanger.

The heart is not only a pump but also a mechanosensory system. We propose that the transduction of the stretch signal involves alteration of potential and intracellular calcium signaling caused by the activation of SACCs in heart cells. It is reasonable to believe that TRP channels, as cellular sensors, may play an important role in this process. As a SACC, TRPC1 functions 
as an element of a mixed cationic $\mathrm{Ca} 2+-$ permeable channel, and the activity of TRPCl may contribute to cardiac MEF. To provide morphological proof in support of this hypothesis, we investigated the expression and distribution of TRPC 1 in the rat hearts. The results showed that mRNA for TRPCl was detected in both the atria and the ventricles. The immunohistochemical study showed that the TRPCl protein is widely expressed in working cardiomyocytes, Purkinje cells, endothelial cells and smooth muscle cells of coronary arterioles, suggesting that TRPCl plays an important role in the rat hearts. The immunofluorescence study revealed a relatively uniform distribution of TRPCl in the surface sarcolemma and T-tubule membrane of ventricular myocytes. There is no transverse-striation pattern of TRPCI in atrial myocytes in accordance with a lack of Ttubules.

Recently it was reported that TRPC1 knockout mouse showed no obvious phenotype, especially store-operated calcium entry in vascular smooth muscle cells (Dietrich et al., 2007). One possible speculation may be the compensatory upregulation of other channels with similar function, which was reported in a study on rats (Selli et al., 2009). Further analysis in different tissues and species should be rewarding. The TRP channels are presumed to be homo- or heterotetramers (Hofmann et al., 2002). The heterologous expression pattern of TRPCl with other endogenous TRP channels in native cells remains to be determined. Functions of TRPC 1 may also be associated with the diversity of channel complexes formed between different isoforms/splice variants and cell-specifically expressed adaptor/signalling proteins. In addition, since the discovery of the TRP channel superfamily, many studies have shown that the TRP superfamily translocate into the plasma membrane upon stimulation (Ambudkar, 2007; Bezzerides et al., 2004; Cayouette and Boulay, 2007) and there is substantial evidence that mechanical stimulation facilitates the membrane trafficking of TRP channels (Inoue et al., 2009). The exact mechanism by which TRP channels insert into the plasma membrane is unknown. Since TRPCl trafficking to the plasma membrane as well as its retention depends on so many factors, it is unclear whether differences in any of these factors can account for the observed discrepancies concerning the issue of channel pheno- types (Gottlieb et al., 2008; Maroto et al., 2005).

The present study has clearly and thoroughly shown the expression and localization pattern of TRPCl in rat hearts in detail and may provide useful data for the future investigations on the functional properties and mechanosensitivity of TRPC1 in rat hearts. The factors involved in regulating TRPCl expression and trafficking as well as the physiological and pathophysiological functions of TRPCl channel in its native environment are worthy of further study.

\section{Acknowledgments}

This research was supported by National Natural Science Foundation of China (30570663, 30770790, 30800377). We thank Xiaobei Zeng and Erjing Gao for providing technical support in carrying out immunohistochemistry and confocal experiments.

\section{References}

Ambudkar IS. Trafficking of TRP channels: determinants of channel function. Handb Exp Pharmacol 2007:541-57.

Antoniotti S, Lovisolo D, Fiorio Pla A, Munaron L. Expression and functional role of $b T R P C l$ channels in native endothelial cells. FEBS Lett 2002;510:189-95.

Bezzerides VJ, Ramsey IS, Kotecha S, Greka A, Clapham DE. Rapid vesicular translocation and insertion of TRP channels. Nat Cell Biol 2004;6:709-20.

Calaghan SC, Belus A, White E. Do stretch-induced changes in intracellular calcium modify the electrical activity of cardiac muscle? Prog Biophys Mol Biol 2003; 82:81-95.

Cayouette $S$, Boulay G. Intracellular trafficking of TRP channels. Cell Calcium 2007;42:225-32.

Clapham DE. TRP channels as cellular sensors. Nature 2003; 426:517-24.

Clemo HF, Stambler BS, Baumgarten CM. Persistent activation of a swelling-activated cation current in ventricular myocytes from dogs with tachycardia-induced congestive heart failure. Circ Res 1998; 83:147-57.

Corey DP, Garcia-Anoveros J, Holt JR, Kwan KY, Lin SY, Vollrath MA, et al. TRPAl is a candidate for the mechanosensitive transduction channel of vertebrate hair cells. Nature 2004;432:723-30.

Dietrich A, Kalwa H, Storch U, Mederos y Schnitzler M, Salanova B, Pinkenburg 0 , et al. Pressure-induced and store-operated cation influx in vascular smooth muscle cells is independent of TRPC1. Pflugers Arch 2007;455:465-77.

Franz MR, Cima R, Wang D, Profitt D, Kurz R. Electrophysiological effects of myocardial stretch and mechanical determinants of stretch-activated arrhythmias. Circulation 1992;86:968-78.

Gottlieb P, Folgering J, Maroto R, Raso A, Wood TG, Kurosky A, et al. Revisiting TRPC1 and TRPC6 mechanosensitivity. Pflugers Arch 2008;455:1097-103.

Hofmann T, Schaefer M, Schultz G, Gudermann T. Subunit composition of mammalian transient receptor potential channels in living cells. Proc Natl Acad Sci USA 2002;99:7461-6.

$\mathrm{Hu} \mathrm{H}$, Sachs F. Stretch-activated ion channels in the heart. J Mol Cell Cardiol 1997; 29:1511-23.

Inoue R, Jian Z, Kawarabayashi Y. Mechanosensitive TRP channels in cardiovascular pathophysiology. Pharmacol Ther 2009.

Iqbal J, Zaidi M. Molecular regulation of mechanotransduction. 
Biochem Biophys Res Commun 2005;328:751-5.

Kwan HY, Huang Y, Yao X. Regulation of canonical transient receptor potential isoform 3 (TRPC3) channel by protein kinase G. Proc Nat Acad Sci USA 2004;101:2625-30.

Liao XD, Tang AH, Chen Q, Jin HJ, Wu CH, Chen LY, et al. Role of $\mathrm{Ca} 2+$ signaling in initiation of stretch-induced apoptosis in neonatal heart cells. Biochem Biophys Res Commun 2003; 310:405-11.

Lin SY, Corey DP. TRP channels in mechanosensation. Curr Opin Neurobiol 2005; 15:350-7.

Liu X, Huang H, Wang W, Wang J, Sachs F, Niu W. Stretch-activated potassium channels in hypotonically induced blebs of atrial myocytes. J Membr Biol 2008;226:17-25.

Maroto R, Raso A, Wood TG, Kurosky A, Martinac B, Hamill OP. TRPCl forms the stretch-activated cation channel in vertebrate cells. Nat Cell Biol 2005;7:179-85.

Minke B, Cook B. TRP channel proteins and signal transduction. Physiol Rev 2002; 82:429-72.

Nilius B, Droogmans G, Wondergem R. Transient receptor potential channels in endothelium: solving the calcium entry puzzle? Endothelium 2003;10:5-15.

Niu W, Sachs F. Dynamic properties of stretch-activated K+ channels in adult rat atrial myocytes. Prog Biophys Mol Biol 2003; 82:12135.

Ohba T, Watanabe H, Murakami M, Takahashi Y, Ino K, Kuromitsu S, et al. Upregulation of TRPCI in the development of cardiac hypertrophy. J Mol Cell Cardiol 2007;42:498-507.

Riccio A, Medhurst AD, Mattei C, Kelsell RE, Calver AR, Randall AD, et al. mRNA distribution analysis of human TRPC family in CNS and peripheral tissues. Brain Res Mol Brain Res 2002;109:95-104.

Sadoshima J, Jahn L, Takahashi T, Kulik TJ, Izumo S. Molecular characterization of the stretch-induced adaptation of cultured cardiac cells. An in vitro model of load-induced cardiac hypertrophy. J Biol Chem 1992a;267:10551-60.

Schrickel JW, Bielik H, Yang A, Schimpf R, Shlevkov N, Burkhardt D, et al. Induction of atrial fibrillation in mice by rapid transesophageal atrial pacing. Basic Res Cardiol 2002;97:452-60.

Selli C, Erac Y, Kosova B, Tosun M. Post-transcriptional silencing of
TRPC1 ion channel gene by RNA interference upregulates TRPC6 expression and store-operated $\mathrm{Ca}(2+)$ entry in A7r5 vascular smooth muscle cells. Vascul Pharmacol 2009.

Shaw A, Xu Q. Biomechanical stress-induced signaling in smooth muscle cells: an update. Curr Vasc Pharmacol 2003;1:41-58.

Tan JH, Liu W, Saint DA. Differential expression of the mechanosensitive potassium channel TREK-1 in epicardial and endocardial myocytes in rat ventricle. Exp Physiol 2004;89:237-42.

Tobita K, Keller BB. Maturation of end-systolic stress-strain relations in chick embryonic myocardium. Am J Physiol Heart Circ Physiol 2000; 279:H216-24.

Vandebrouck C, Martin D, Colson-Van Schoor M, Debaix H, Gailly P. Involvement of TRPC in the abnormal calcium influx observed in dystrophic $(\mathrm{mdx})$ mouse skeletal muscle fibers. J Cell Biol 2002; 158:1089-96.

Wei $H$, Huang $H X$, Wang $W$, Zhang ZF, Fu XS, Liu P, et al. A ventricular pressure-clamping system for the study of mechano-electrical feedback. Sheng Li Xue Bao 2006;58:606-10.

Wes PD, Chevesich J, Jeromin A, Rosenberg C, Stetten G, Montell C. TRPC1, a human homolog of a Drosophila store-operated channel. Proc Natl Acad Sci USA 1995;92:9652-6.

Xian Tao L, Dyachenko V, Zuzarte M, Putzke C, Preisig-Muller R, Isenberg $G$, et al. The stretch-activated potassium channel TREK-1 in rat cardiac ventricular muscle. Cardiovasc Res 2006;69:86-97.

Yip H, Chan WY, Leung PC, Kwan HY, Liu C, Huang Y, et al. Expression of TRPC homologs in endothelial cells and smooth muscle layers of human arteries. Histochem Cell Biol 2004;122:55361.

Zhu X, Chu PB, Peyton M, Birnbaumer L. Molecular cloning of a widely expressed human homologue for the Drosophila trp gene. FEBS Lett 1995;373:193-8.

Zitt C, Zobel A, Obukhov AG, Harteneck C, Kalkbrenner F, Luckhoff A, et al. Cloning and functional expression of a human $\mathrm{Ca}$ 2+-permeable cation channel activated by calcium store depletion. Neuron 1996; 16:1189-96.

Zou H, Lifshitz LM, Tuft RA, Fogarty KE, Singer JJ. Visualization of $\mathrm{Ca} 2+$ entry through single stretch-activated cation channels. Proc Natl Acad Sci USA 2002;99:6404-9. 
H. Huang et al. 